\title{
Estudio de repetibilidad por medio de desviación estándar de un aberrómetro ocular de tipo Shack - Hartmann
}

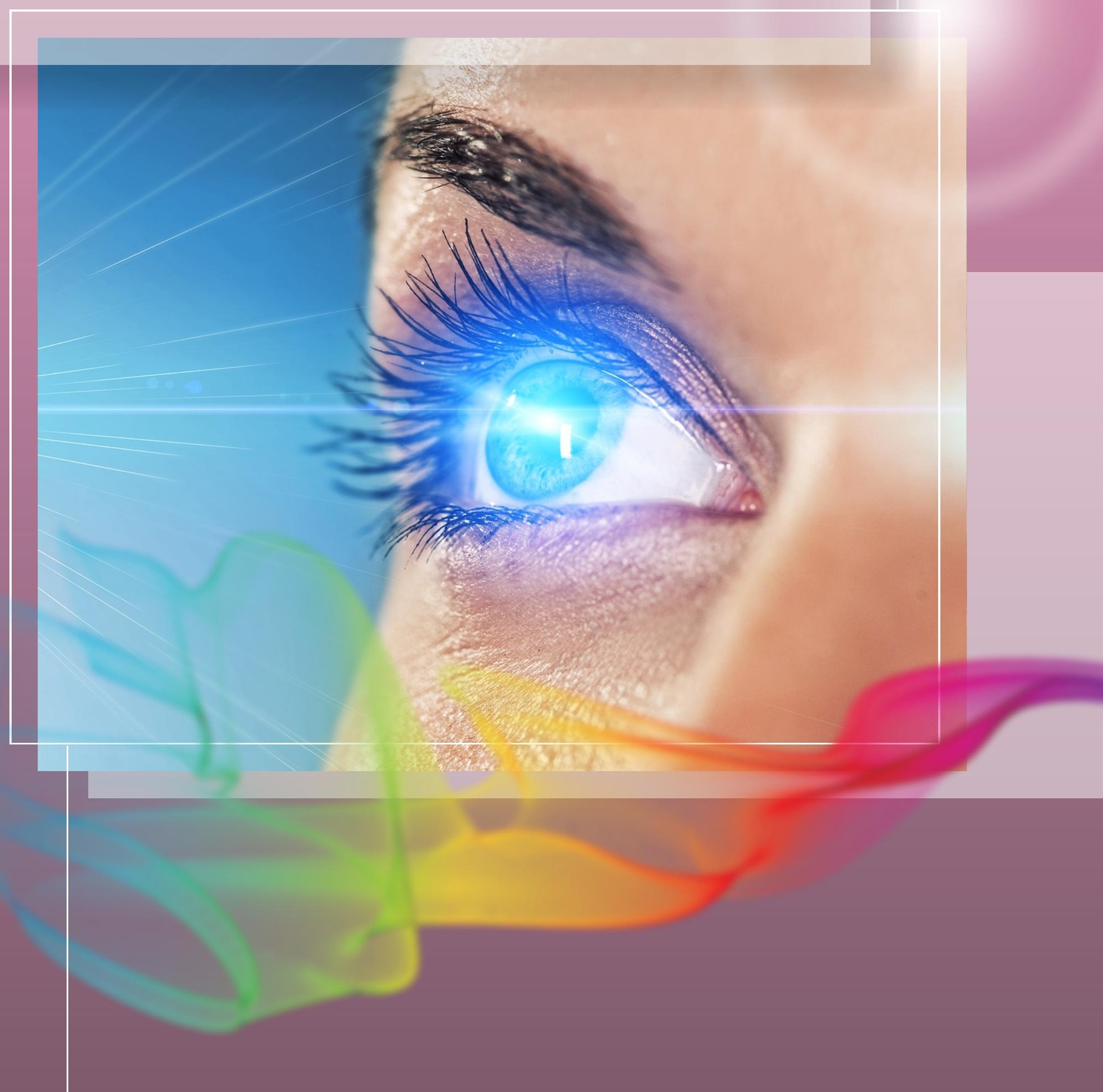




\title{
Estudio de repetibilidad por medio de desviación estándar de un aberrómetro ocular de tipo Shack - Hartmann'. Repeatability study by standard deviation of a Shack - Hartmann type aberrometer
}

\author{
Luis Gabriel Valdivieso González ${ }^{2}$, Andrea Fernanda Muñoz Potosí, Sandra Eloisa \\ Balderas Mata ${ }^{4}$, Eduardo Tepichín Rodríguez. \\ ${ }^{2,3,5}$ Instituto Nacional de Astrofísica, Óptica y Electrónica, San Andrés Cholula, México. \\ ${ }^{4}$ Universidad de Guadalajara, Guadalajara, Jalisco, México.
}

Artículo recibido en agosto de 2015; artículo aceptado en noviembre de 2015.

Citación del artículo: L. G. Valdivieso González, A. F. Muñoz-Potosí, S. E. Balderas-Mata, E. Tepichín Rodríguez (2015). "Análisis de repetibilidad en la medición para un arreglo experimental de aberrómetro ocular,"I+D Revista de Investigaciones, 6(2), 48-56.

\section{Resumen}

La configuración habitual de un arreglo para medir las aberraciones oculares, utiliza un haz infrarrojo, el cual es focalizado en la retina con el fin de que actúe como una fuente secundaria de radiación. La luz reflejada emerge a través del sistema óptico del ojo, modulada con las aberraciones totales. Este frente de onda aberrado se mide con un sensor de tipo Shack - Hartmann.

\footnotetext{
${ }^{1}$ Artículo de investigación, de enfoque cuantitativo, resultado de un proyecto de investigación culminado, perteneciente al área de la Física, subárea Óptica, desarrollado en el grupo de Ciencias de la Imagen y Física de la Visión, fue financiado por el Consejo Nacional de Ciencia y Tecnología (México) con el proyecto número 98777 PY. Fecha de inicio: 2010, fecha de terminación: 2014.

${ }^{2}$ Físico, Universidad Industrial de Santander. Magíster en Física, Universidad Industrial de Santander, Doctor en Ciencias con especialidad en Óptica, Instituto Nacional de Astrofísica, Óptica y Electrónica. Docente-investigador del grupo FIELDS. Universitaria de Investigación y Desarrollo (UDI) de la ciudad de Bucaramanga (Colombia). Dirección: Calle 9 No.23-55, PBX6352525.Correo electrónico institucional: gabriel.valdivieso@inaoep.mx

${ }^{3}$ Ingeniera Física, Universidad del Cauca. Maestra en Ciencias con especialidad en Óptica, Instituto Nacional de Astrofísica, Óptica y Electrónica. Estudiante de Doctorado en Ciencias con especialidad en Óptica, Instituto Nacional de Astrofísica, Óptica y Electrónica de la ciudad de San Andrés Cholula (México). Dirección: Calle Luis Enrique Erro No. 1. Correo electrónico institucional: amunozpotosi@inaoep.mx

${ }^{4}$ Licenciada en Física, Universidad Autónoma de San Luis Potosí. Maestra en Ciencias con especialidad en Óptica, Instituto Nacional de Astrofísica, Óptica y Electrónica. Doctora en Ciencias con especialidad en Óptica, Instituto Nacional de Astrofísica, Óptica y Electrónica. Posdoctorado en Óptica Visual, University of California, Davis. Docente-investigador del grupo de Instrumentación, Óptica, Electrónica y Fotónica, Centro Universitario de Ciencias Exactas e Ingenierías, Universidad de Guadalajara. Dirección: Blvd. Marcelino García Barragán No. 1421, C.P. 44430. Correo electrónico institucional: sandra.balderas@cucei.udg.mx

${ }^{5}$ Ingeniero Físico, Universidad Iberoamericana, México D.F. Maestro en Ciencias con especialidad en Óptica, Instituto Nacional de Astrofísica Óptica y Electrónica, Puebla, México y doctor en Física, con especialidad en Óptica, Instituto Nacional de Astrofísica Óptica y Electrónica Puebla, México. Dirección: Calle Luis Enrique Erro No. 1. Correo electrónico institucional: tepichini@inaoep.mx.
} 
Un aberrómetro ocular es colocado en lazo cerrado con un espejo deformable para ensamblar un arreglo experimental de óptica adaptativa (AO), el cual es usado en instrumentos oftalmológicos para mejorar su resolución lateral. En este trabajo se presenta el análisis de repetibilidad en las mediciones para un sistema experimental que permite obtener las aberraciones de un ojo humano in-vivo, el mismo que posteriormente será usado como parte de un sistema de óptica adaptativa de un arreglo experimental de fondo de ojo.

Palabras clave: repetibilidad, aberrómetro, polinomios de Zernike, óptica adaptativa.

\section{Abstract}

The usual configuration for a system that measures ocular aberrations uses an infrared beam, which is focused on the retina, in order to act as a secondary source of radiation. The back reflected light from the retina emerges through the eye's optical system modulated with the total aberrations. This aberrated wavefront is measured with a Shack-Hartmann type sensor. An ocular aberrometer, could be connected, in a closed loop, with a deformable mirror in order to assemble an experimental setup of adaptive optics (AO). It is useful in ophthalmic instruments because improves the lateral resolution. In this work, the analysis of repeatability of the measurements for an experimental setup that allows measuring the aberrations of an in-vivo human eye is shown. This system will be used as part of an adaptive optics system for a fundus camera.
Keywords: repeatability, aberrometer, Zernike polynomials, adaptive optics.

\section{Introducción}

En los sistemas ópticos formadores de imagen existen imperfecciones que producen una imagen borrosa, las cuales son conocidas como aberraciones. Estas provocan que la imagen producida no represente fielmente el objeto observado. Las aberraciones pueden dividirse según su causa en aberraciones geométricas o monocromáticas y aberraciones cromáticas.

En este trabajo el interés se centra en las aberraciones geométricas, las cuales son causadas por imperfecciones en la forma $y$ colocación de los elementos de un sistema óptico. El ojo humano, como sistema formador de imágenes, presenta por naturaleza este tipo de imperfecciones, las cuales representan un problema cuando se intenta obtener una imagen de fondo de ojo, en particular de la retina.

A mediados de la década de los 90, Junzhong Liang (1994) utilizó la técnica de Shack Hartmann para medir aberraciones monocromáticas de ojos humanos. Desde entonces, varios autores han desarrollado $\mathrm{y}$ probado arreglos experimentales para la medida de aberraciones usando esta técnica con el fin de aplicarla para su uso en clínica (Thibos L. \& Hong X., 1999, y Cheng X., Himebaugh N., Kollbaum P., Thibos L. \& Bradley A., 2003). Recientemente Fedtke, C., Ehrmann K., Falk D. \& Holden B. (2012) publicaron resultados de la validación en la medida de aberraciones en diferentes puntos de la pupila . 
En este trabajo se presenta una descripción del arreglo experimental implementado para la medida de aberraciones de ojos humanos in-vivo, el cual será usado como el brazo de óptica adaptativa de un sistema de captura de fondo de ojo. Se presentan los resultados obtenidos para las aberraciones intrínsecas del arreglo experimental analizando la repetibilidad de las mediciones.

\section{Arreglo experimental}

\section{Descripción del arreglo}

Un arreglo experimental para la obtención de las aberraciones en el ojo humano consiste en hacer incidir sobre la córnea un haz colimado con el fin de enfocarlo sobre la retina, produciendo una mancha circular asociada con la difracción de Airy. El punto generado actúa como una fuente secundaria de radiación que recorre el sistema óptico del ojo recogiendo las aberraciones de este. Dicho haz aberrado se conduce por medio de un sistema óptico hacia un sensor de frente de onda (WFS) de tipo Shack-Hartmann.

En este tipo de sensor la medición de las aberraciones se lleva a cabo mediante la colocación de una matriz bidimensional de pequeñas lentes en un plano conjugado con la pupila del sistema y un sensor CCD en el plano focal posterior de las lentillas, como se muestra en la Figura 1. Determinando el desplazamiento de los puntos imagen, generados por las lentillas sobre el sensor tipo CCD, respecto a los correspondientes para un frente de onda plano, es posible calcular la curvatura local del frente de onda.

Figura 1. Principio básico del sensor de frente de onda de tipo Shack-Hartmann

Frente de onda plano

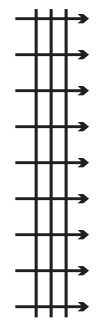

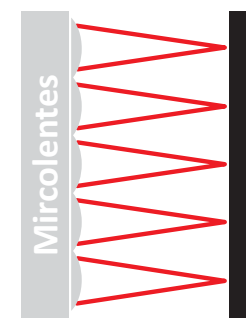

Sensor CCD
Frente de onda aberrado
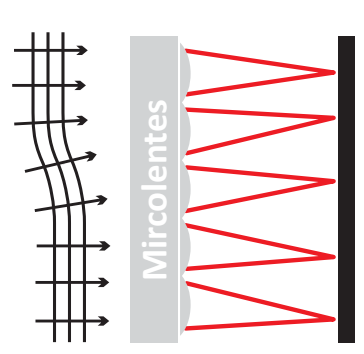

Sensor CCD

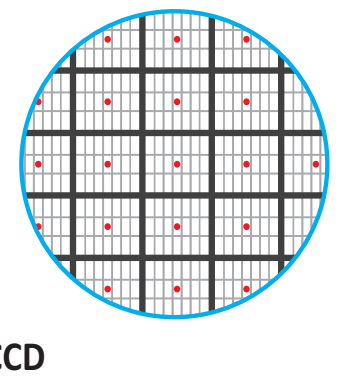

Fuente: Los autores.

La aberración del frente de onda del haz proveniente del ojo se calcula en términos de los polinomios de Zernike, los cuales son una base orto-normal de polinomios definidos sobre un disco de radio unitario, cada uno de los cuales identifica una de las aberraciones del arreglo, como son defoco y astigmatismo, entre otras. De esta manera, los coeficientes necesarios para reconstruir el frente de onda representan el valor de cada aberración presente en el sistema óptico.

La aberración medida será posteriormente compensada por medio de óptica adaptativa, e.g., usando un espejo deformable (DM), lo cual 
permitirá obtener imágenes de fondo de ojo con buena resolución lateral cuando el aberrómetro haga parte del sistema de óptica adaptativa en el arreglo experimental de captura de fondo de ojo.

En este trabajo se utiliza una configuración reducida para el arreglo experimental de captura de imágenes in vivo del fondo de ojo, mediante la reducción en el número de lentes en comparación con la configuración usada por Balderas-Mata E., Jones S. M., Zawadski R. J. \& Werner J. S. (2011). De este modo, se reducen las aberraciones propias del arreglo y se facilita su alineación.

El arreglo experimental propuesto para medir las aberraciones del ojo humano se muestra en la Figura 2. En este se utilizan dos sistemas telescópicos de tipo Kepler, conformados por las parejas de lentes positivas L1-L2 y L3-L4, encargados de reescalar el diámetro del frente de onda colimado de acuerdo con el diámetro de pupila en cada sección del arreglo.

Figura 2. Diagrama del arreglo experimental propuesto

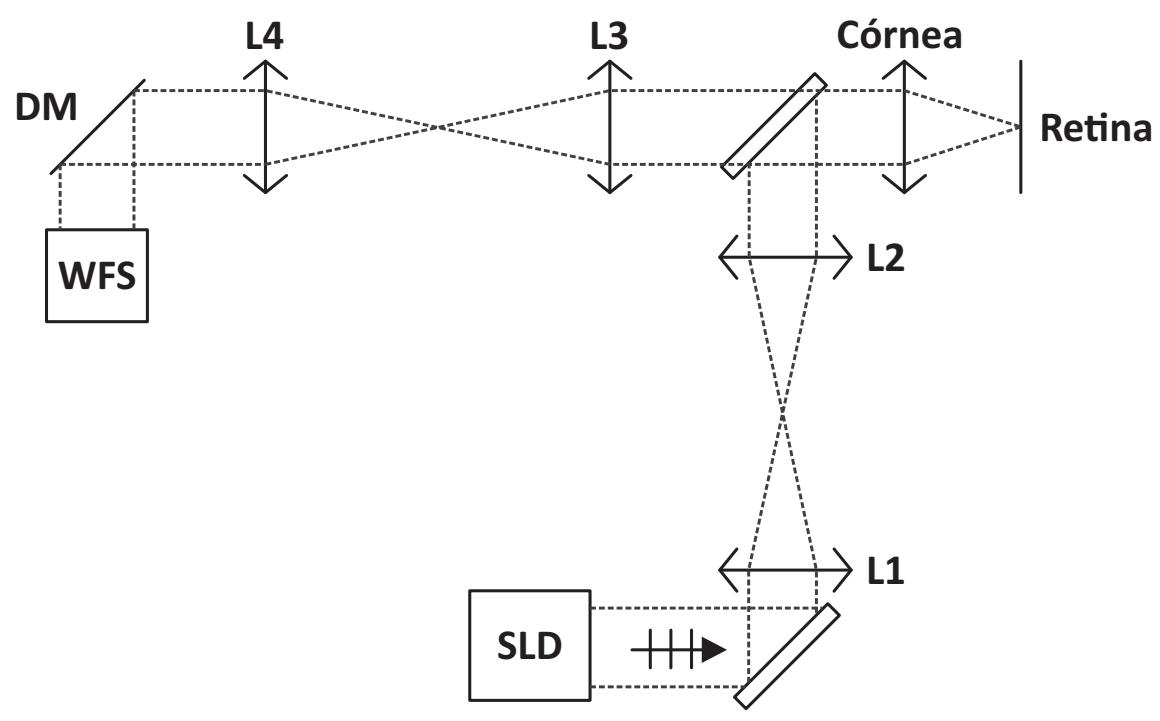

Fuente: Los autores.

Es importante considerar que la fuente de iluminación utilizada en el arreglo fue un diodo súper luminiscente (SLD), con una longitud de onda de $830(\mathrm{~nm})$ y un ancho de banda de 20 (nm).

\section{Medida de las aberraciones propias del arreglo}

Antes de realizar mediciones con el sistema es importante conocer su mapa de aberración intrínseco. Para ello, se ubica un espejo planoparalelo en la posición de la córnea (ver Figura 2). Dado que el espejo plano se considera sin aberraciones, los coeficientes en los polinomios de Zernike que se obtengan con el sensor de frente de onda serán causados únicamente por el sistema óptico i.e. aberrómetro.

En un arreglo experimental para aberrómetro ideal, el mapa de aberraciones esperado 
corresponde al de un frente de onda plano. Sin embargo, en un sistema real pueden esperarse pequeñas diferencias. En la Figura 3 se muestra el mapa de aberraciones del sistema óptico, graficado en términos de los polinomios de Zernike.

Figura 3. Aberraciones intrínsecas del sistema. a) Mapas de aberraciones b) Coeficientes de los polinomios de Zernike
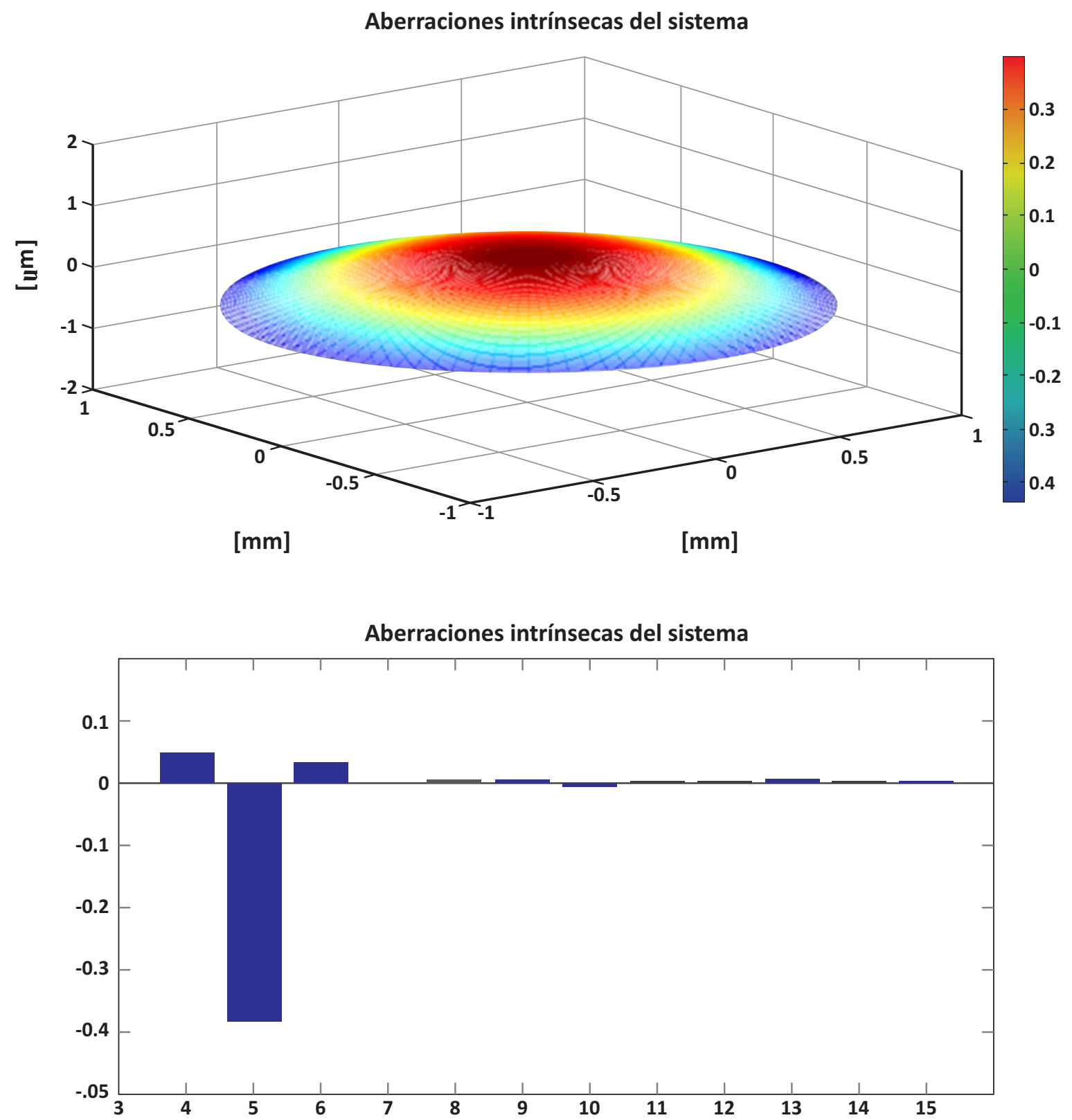

Número de Coeficiente

Fuente: Los autores. 
Conocer el mapa de aberración intrínseco del arreglo es de utilidad, ya que corresponde a un error sistemático que se puede restar algebraicamente de las mediciones posteriores. Realizar esta medición a diferentes horas del día, durante varios días, permitirá determinar la repetibilidad en las mediciones usando el arreglo experimental descrito en la Figura 2.

\section{Resultados experimentales}

El sistema se caracterizó en términos de la repetibilidad en las mediciones, las cuales pueden variar debido al cambio en la temperatura, lo cual afectaría la alineación del sistema. Dicha caracterización se realizó midiendo en repetidas ocasiones el mapa de aberraciones propio del arreglo.

En la Figura 4 se presentan los resultados obtenidos para los primeros tres órdenes de coeficientes de los polinomios de Zernike, para la primera y última de las repeticiones.

Figura 4. Comparación de los resultados obtenidos en la prueba de repetibilidad. a) Coeficientes para la primera medición. b) Coeficientes para la última medición.

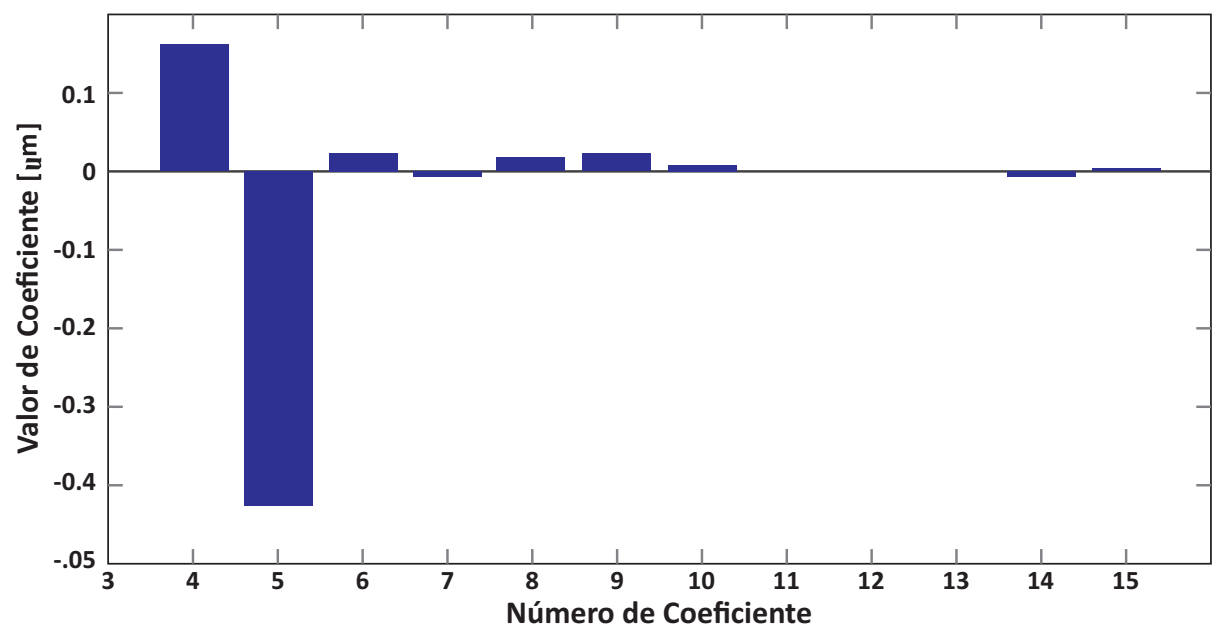

(a)

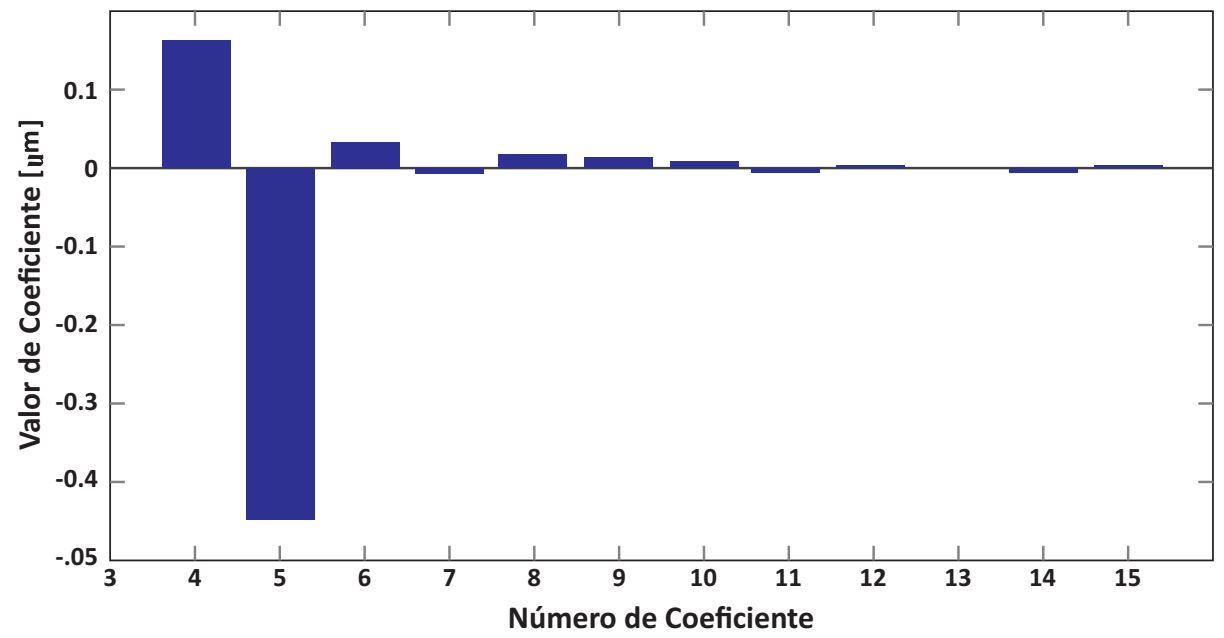

(b)

Fuente: Los autores. 
Con el fin de determinar la variación de los coeficientes de los polinomios de Zernike con el tiempo, se calculó su promedio y desviación estándar, cuyos resultados se presentan en la Tabla 1.

Los tres primeros coeficientes de los polinomios de Zernike no se tuvieron en cuenta en este trabajo, ya que corresponden a los polinomios que representan las aberraciones de pistón y tilt en las direcciones $\mathrm{x}$ e y del plano de observación, asociados con errores mecánicos o de alineación, los cuales no aportan información sobre la calidad del sistema formador de imagen que se desea medir.

Tabla 1. Promedio y desviación estándar para los coeficientes de los polinomios de Zernike

Los resultados demuestran que las variaciones de todos los coeficientes son muy pequeñas, tal que

\begin{tabular}{ccc}
\hline Coeficiente & Promedio $(\boldsymbol{\mu m})$ & Desviación std $(\boldsymbol{\mu m})$ \\
\hline $4(2,-2)$ & 0.174 & 0.006 \\
$5(2,0)$ & -0.426 & 0.011 \\
$6(2,2)$ & 0.014 & 0.011 \\
$7(3,-3)$ & -0.010 & 0.006 \\
$8(3,-1)$ & 0.019 & 0.005 \\
$9(3,1)$ & 0.013 & 0.002 \\
$10(3,3)$ & 0.006 & 0.002 \\
$11(4,-4)$ & -0.002 & 0.001 \\
$12(4,-2)$ & 0.001 & 0.001 \\
$13(4,0)$ & 0.003 & 0.001 \\
$14(4,2)$ & 0.002 & 0.001 \\
$15(4,4)$ & 0.004 & 0.001 \\
\hline
\end{tabular}

pueden despreciarse en el momento de hacer las mediciones.

Los coeficientes de mayor interés en este estudio son el desenfoque, correspondiente al quinto polinomio de Zernike, y las dos orientaciones principales del astigmatismo, correspondientes a los polinomios de Zernike cuarto y sexto, ya que son los que el optómetra utiliza para recetar los lentes de corrección a sus pacientes.

\section{Conclusiones}

Se midieron las aberraciones intrínsecas del arreglo experimental para la medida de aberraciones de ojos humanos, con lo cual se tiene información acerca del error sistemático que estará incluido en todas las mediciones. Este valor, aunque pequeño, se tendrá en cuenta en el momento de reportar la medición aberraciones de ojos humanos, mejorando la precisión de los resultados

Se caracterizó un arreglo experimental para la 
medida de aberraciones de ojos humanos a partir de la repetibilidad en la medición de las aberraciones propias del arreglo. Se probó que el arreglo experimental presenta resultados con muy alta repetibilidad cuando se comparan los datos medidos usando la desviación estándar

\section{Agradecimientos}

Esta investigación es apoyada por el Consejo Nacional de Ciencia y Tecnología CONACyT de México, mediante el proyecto número $98777 \mathrm{PY}$.

\section{Referencias}

Balderas Mata S. E., Jones S. M., Zawadski R. J. \& Werner J. S. (2011). Application of a new hispeed magnetic deformable mirror for in-vivo retinal imaging. Proceedings of 22nd Congres of the International Commission for Optics, $80111,8011195-1-8011955-8$ Doi: $10.1117 / 12.903365$
Cheng X., Himebaugh N., Kollbaum P., Thibos L. \& Bradley A. (2003). Validation of a Clinical Shack-Hartmann Aberrometer. Optometry and Vision Science, 80,587-595. Doi:10.1097/00006324-200308000-00013

Fedtke C., Ehrmann K., Falk D. \& Holden B. (2012). Validation of a quasi real-time global aberrometer: The eyemapper. Proceedings of SPIE Photonics West 2012, 8209,82090C-182090C-7. Doi:10.1117/12.906927

Liang J., Grimm B., Goelz S. \& Bille J.F. (1994). Objective measurement of wave aberrations of the human eye with the use of a Hartmann-Shack wave-front sensor. Journal of Optical Society of America, 11, 1949-1957. Doi:10.1364/JOSAA.11.001949

Thibos L. \& Hong X. (1999). Clinical Applications of the Shack-Hartmann Aberrometer. Optometry and Vision Science, $76,817-825$. 